\title{
MECHANICAL OUTPUT ABOUT THE ANKLE IN COUNTERMOVEMENT JUMPS AND JUMPS WITH EXTENDED KNEE *
}

\author{
J.B. de GRAAF, M.F. BOBBERT, W.E. TETTEROO \\ and G.J. van INGEN SCHENAU
}

Free University of Amsterdam, The Netherlands

De Graaf, J.B., M.F. Bobbert, W.E. Tetteroo and G.J. van Ingen Schenau, 1987. Mechanical output about the ankle in countermovement jumps and jumps with extended knee. Human Movement Science $6,333-347$.

In this study the mechanical output (e.g., force, contraction velocity, instantaneous power) about the ankle was measured during a jump with and without occurrence of transportation of power and pre-stretch potentiation. To examine this, a model of the $\mathrm{m}$. triceps surae was used. Eleven subjects performed a maximal one-legged countermovement jump (CMJ) and a maximal one-legged Jump with extended knee (EKJ). Ground reaction forces, cinematographic data and electromyograms of $\mathrm{m}$. triceps surae were recorded. The power output, defined as the product of moment and angular velocity, and work done about the ankle were higher during CMJ (1404 W, $130 \mathrm{~J})$ than during EKJ ( $852 \mathrm{~W}, 76 \mathrm{~J}$ ). The peak moments were the same during both jumps. The model of the $\mathrm{m}$. triceps surae showed that the power delivered by $\mathrm{m}$. triceps surae was higher during CMJ than during EKJ, as a result of catapult-action of $\mathrm{m}$. gastrocnemius tendon. The difference in work done is explained, in addition to transportation of energy, by a greater contraction range of $\mathrm{m}$. soleus during CMJ. There is no reason to assume that pre-stretch potentiation plays a role in the difference in mechanical output. The resuits show that the mechanical output of muscles in complex movements is strongly dependent on the dynamics of the movement, and not only on its contractile capacity.

\section{Introduction}

In previous studies (Bobbert et al. 1985; Van Ingen Schenau et al. 1985) was shown that the mechanical output of muscles during a

\footnotetext{
* Requests for reprints should be sent to J.B. de Graaf, Dept. of Functional Anatomy, Faculty of Human Movement Sciences, Free University of Amsterdam, P.O. Box 7161, 1007 MC Amsterdam, The Netherlands.
} 
complex movement, as the vertical jump, is difficult to explain on the basis of muscle properties. Power output found about the ankle (defined as the product of net moment and angular velocity) during plantar flexion in one-legged jumping has been found to reach a peak value of $1800 \mathrm{~W}$ (Van Soest et al. 1985). This is much more than the $214 \mathrm{~W}$ registered during isokinetic plantar flexion (Fugl-Meyer et al. 1982), and also much more than the maximum power output (175 W) predicted from the dimensions and architecture of plantar flexors of human cadaver specimens (Woittiez et al. 1985). The discrepancies between power output about the ankle during jumping and power output of the plantar flexors predicted from dimensions and architecture can be attributed to several mechanisms. The first mechanism is liberation of energy from tendinous structures, which occurs when the force exerted on these structures decreases. This phenomenon is called catapult-action (Alexander and Bennet-Clark 1977; Hof et al. 1983; Van Ingen Schenau et al. 1985). The second mechanism is transportation of power from knec to ankle via the bi-articular m. gastrocncmius (Fugl-Meyer et al. 1982; Van Ingen Schenau 1984). This transport means that part of the power found about the ankle, as the product of moment and angular velocity, is actually delivered by the extensors of the knee, and is transported from knee to ankle by the tendinous like action (Elftman 1939) of the $\mathrm{m}$. gastrocnemius. A third factor, mentioned in the literature, is pre-stretch potentiation (Cavagna 1978). In jumping, the calf muscles are stretched during the countermovement. It is assumed that stretching of an active muscle enhances the capability of the contractile elements to deliver force, power and work.

The purpose of this study is to gain more insight into the relation between fundamental muscle mechanics and the mechanical output in complex multi-articular movements. For this purpose the mechanical output about the ankle in a one-legged countermovement jump is compared with that in a one-legged jump with extended knee. During jumping with extended knee $\mathrm{m}$. gastrocnemius cannot transport any power from the knee to the ankle (as the angular velocity in the knee remains zero) and pre-stretch of the plantar flexors does not occur. It is attempted to explain the difference in mechanical output between the two types of jumps with the help of a model of the muscle-tendon complex of $\mathrm{m}$. triceps surae. The model allows for calculation of the mechanical output of contractile elements and series elastic elements (tendon) of both $\mathrm{m}$. soleus and $\mathrm{m}$. gastrocnemius. 


\section{Methods}

\section{Subjects and experimental protocol}

Eleven healthy subjects (age $23.4 \pm 2.8 \mathrm{yr}$, height $1.83 \pm 0.07 \mathrm{~m}$, body mass $75.6 \pm 5.5 \mathrm{~kg}$ ) participated in this study. In jumping with extended knee, angular movement in the hip was allowed. The subjects practiced the performance of the two types of jump until they were able to execute them satisfactorily. After this practice they performed five one-legged vertical jumps with preparatory countermovement (CMJ) and five one-legged vertical jumps with extended knee (EKJ). In both jumps the subjects were instructed to keep their hands akimbo, to keep the position of the contralateral leg constant and to jump as high as they could.

\section{Kinematics and kinetics}

As described previously (Van Soest et al. 1985) anatomical landmarks were placed on the skin in order to define the positions of foot, lower leg, upper leg and upper body. During jumping, the subjects were filmed in the sagittal plane ( $16 \mathrm{~mm}$ film, 100 frames $/ \mathrm{sec})$, and vertical and fore-aft components of the ground reaction force, as well as the center of pressure under the foot, were measured by means of a force platform (KISTLER type 9281B). For each frame, coordinates of the landmarks were determined with the help of a motion analyzer (DYNAMIC FRAME). Absolute coordinates obtained after proper scaling were low-pass filtered (Butterworth 4th order zero lag filter, cutoff frequency of $16 \mathrm{~Hz}$ ) and numerical differentiation with respect to time yielded velocities of the anatomical landmarks. Subsequently, joint angles, angular velocities and angular accelerations, and the vertical velocity difference between the mass center of body and the ankle $\left(\dot{Y}_{\mathrm{MCB}}-\dot{Y}_{\mathrm{A}}\right)$ were calculated. After synchronization of film and force platform data the resultant moment about the ankle was calculated by solving equations concerning a free body diagram of the foot according to Elftman (1939).

In order to obtain for $\mathrm{m}$. gastrocnemius and $\mathrm{m}$. soleus $\Delta L_{O I}$, the distance between origin and insertion relative to a reference distance (the distance when the knee is flexed $90^{\circ}$ and the sole of the foot is at right angles to the shank) a model devised by Grieve et al. (1978) was 
used. This model was also used to calculate moment arms of m. soleus and $\mathrm{m}$. gastrocnemius at the ankle, and the moment arm of $\mathrm{m}$. gastrocnemius at the knee. The velocities with which origins of the muscles approach insertion $\left(V_{O I}\right)$ was acquired by numerical differentiation of $\Delta L_{O I}$. Shortening of the muscle was defined positive.

\section{Electromyography}

After standard skin preparation techniques (Basmajian 1978), pairs of surface electrodes were placed in longitudinal direction on the skin covering $\mathrm{m}$. soleus and medial and lateral heads of $\mathrm{m}$. gastrocnemius. The positioning of electrodes is described by Gregoire et al. (1984). The electrical signals were amplified (DISA type 15 C01), high-pass filtered (cutoff frequency $=10 \mathrm{~Hz}$ ), full wave rectified and integrated $(-3 \mathrm{~dB}$ at $5 \mathrm{~Hz}$, slope $6 \mathrm{~dB} /$ octave), and sampled simultaneously with the ground reaction force. During both jumps the resulting values of EMG-level of each muscle were expressed in terms of percent of the maximum value attained during $\mathrm{CMJ}$.

\section{Treatment of data}

The highest CMJ and the highest EKJ of each subject were selected for analysis. The start of the push-off phase was defined as the instant that the mass center of the body began to move upward. Mean curves ( $\pm S E M$, Standard Error of Means) of variables were calculated for the group of subjects after synchronization of individual curves on the instant the toes lost contact with the force platform.

\section{Model of the muscle-tendon complex of $m$. triceps surae}

The model, which has been set out in detail elsewhere (Bobbert et al. 1986), will be described briefly here. It is composed to two units in parallel. One unit represents the muscle-tendon complex of $\mathrm{m}$. soleus, the other represents that of $\mathrm{m}$. gastrocnemius. The input of the model consists of the time histories of $V_{O I}$. The choice of parameter values was based on assumptions concerning muscle dimensions, muscle architecture, and physiological behavior of muscle fibers, as well as on assumptions concerning dimensions and elastic behavior of tendinous structures in series with the muscle fibers. These assumptions have all 


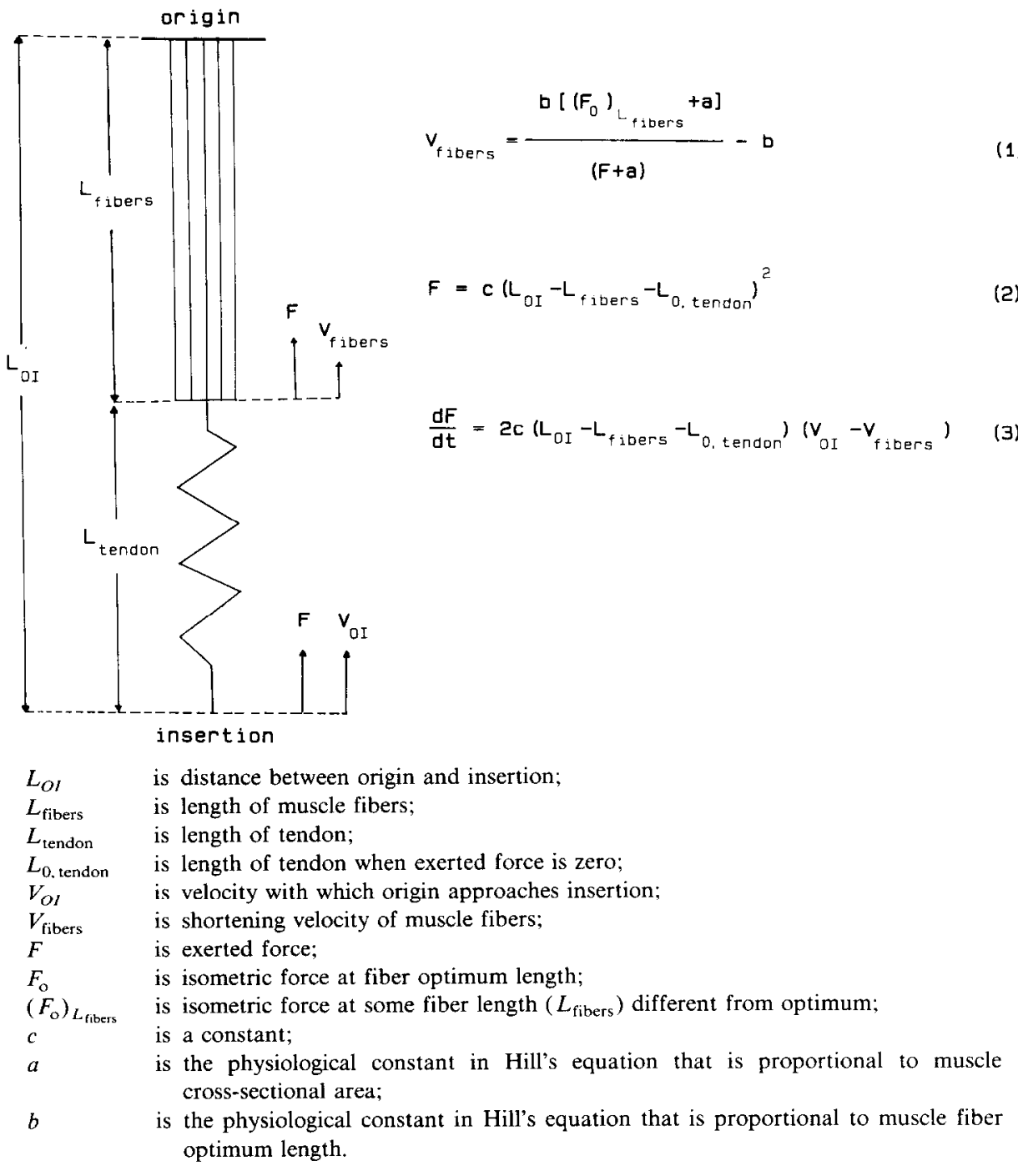

Fig. 1. Model developed of a muscle-tendon complex, and equations used for describing force-velocity relationship of muscle fibers (eq. (1)), force-elongation relationship of tendinous tissue (eq. (2)) and a relationship between rate of change of force and rate of change of tendon length, the latter being equal to the difference between $V_{O I}$ and $V_{\text {fibers }}$ (eq. (3)).

been detailed in a previous paper (Bobbert et al. 1986). The model presupposes a constant level of muscle excitation.

The procedure for studying the mechanical output of each unit 
during jumping is as follows. First an equilibrium situation is defined at zero $V_{O I}$. Subsequently, the mean time histories of $V_{O I}$ are imposed upon the unitstarting from the instant that $V_{O I}$ becomes positive. Shortening velocity of muscle fibers $\left(V_{\text {fibers }}\right)$, exerted force $\left(F_{\text {Inudel }}\right)$ and shortening velocity of tendon $\left(V_{\text {tendon }}\right)$ were calculated with help of the equations illustrated in fig. 1 . Integrating $V_{\text {fibers }}$ and $V_{\text {tendon }}$ with respect to time gives length of muscle fibers ( $L_{\text {fibers }}$ ) and elongation of tendon $\left(\Delta L_{\text {tendon }}\right)$, respectively. By multiplication of $V_{\text {tendon }}$ and $F_{\text {model }}$ the power output of tendon ( $\left.P_{\text {tendon }}\right)$ is obtained. Multiplication of $V_{\text {fibcrs }}$ and $F_{\text {model }}$ yields the power output of the muscle fibers $\left(P_{\text {fibers }}\right)$. The power transported from knee to ankle ( $P_{\text {transported }}$ ) was obtained by multiplication of the moment exerted by $\mathrm{m}$. gastrocnemius about the knee (given by the product of calculated force and moment arm at the knee) and angular velocity of knee extension. In situations where the total muscle-tendon complex of $\mathrm{m}$. gastrocnemius shortens (as is the case in this study) this flow of energy from knee extensors to $\mathrm{m}$. gastrocnemius is used to reinforce plantar flexion.

The starting length of the muscle fibers of both muscles in CMJ was selected to be $6.6 \mathrm{~cm}$ (corresponding to $110 \%$ of fiber optimum length) (Bobbert et al. 1986). In EKJ the starting length of muscle fibers of $\mathrm{m}$. soleus was only $5 \mathrm{~cm}$. This appeared to be the equilibrium situation at the $\Delta L_{O I \text {, start }}$ in this jump. The reason for the reduction of starting length is that the ankle angle is greater in EKJ than in CMJ at the start of plantar flexion, so m. soleus is shorter in EKJ. The starting length of muscle fibers of $\mathrm{m}$. gastrocnemius is the same in both jumps, because in EKJ the knee angle is also greater at the start of plantar flexion.

\section{Results}

The mean time histories of net moments about the ankle $\left(M_{\mathrm{A}}\right)$ for the two types of jumps are shown in fig. 2. The peak moment is $194 \pm 28 \mathrm{Nm}$ for CMJ, and $193 \pm 28 \mathrm{Nm}$ for EKJ. The rise in moment is accompanied by a rise in EMG-levels as can be seen in fig. 3. Note that the EMG-levels of the calf muscles start to decrease earlier in EKJ than in CMJ. Bearing in mind that there is a delay time of approximately $50 \mathrm{msec}$ between EMG and mechanical output (Van Soest et al. 1985), this finding indicates that during the last part of the push-off muscle-excitation during EKJ is not constant but decreasing. 

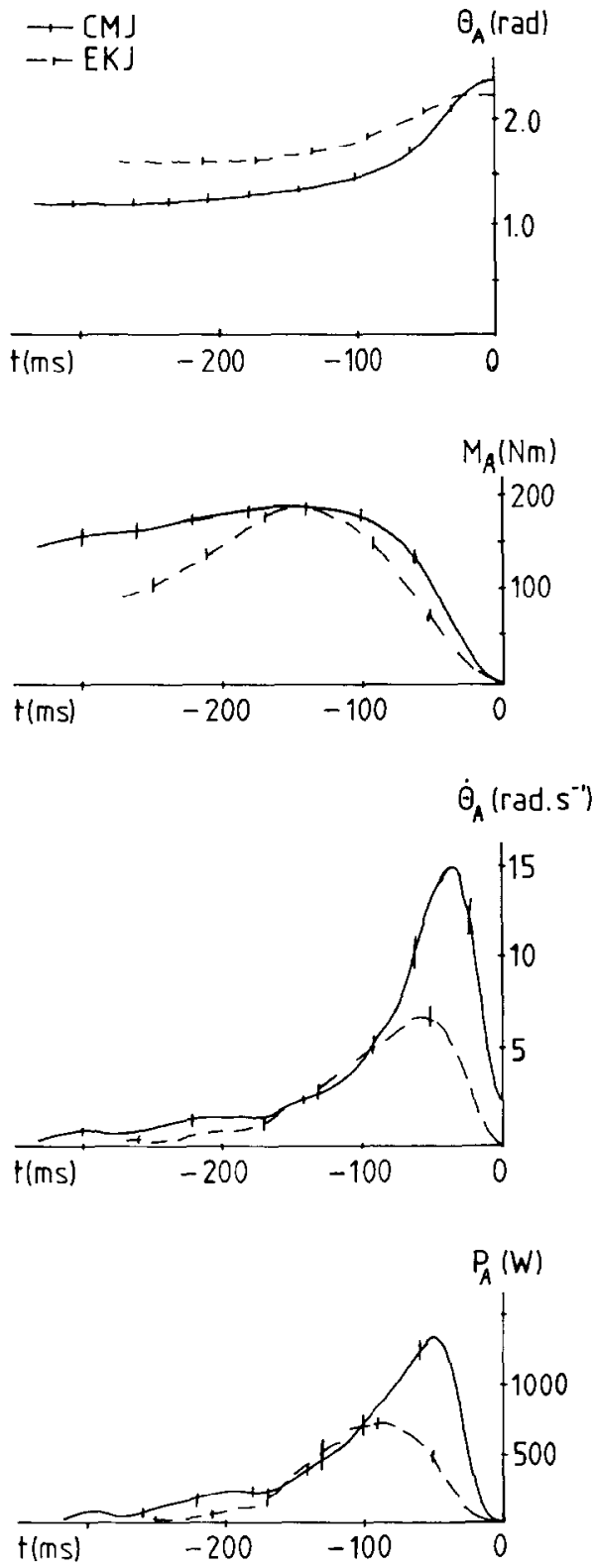

Fig. 2. Mean time histories of angle in the ankle $\left(\Theta_{\mathrm{A}}\right)$, moment about the ankle $\left(M_{\mathrm{A}}\right)$, angular velocity of plantar flexion $\left(\dot{\Theta}_{\mathrm{A}}\right)$, and power output about the ankle $\left(P_{\mathrm{A}}\right)$ for a one-legged countermovement jump (CMJ) and a one-legged jump with extended knee (EKJ). Time $(t)$ is expressed relative to instant of toe-off $(t=0)$. Thin vertical bars indicate SEM. $n=11$. 

$-[M]$
EMG-level
-- EKJ
(\% of max. (MJ)

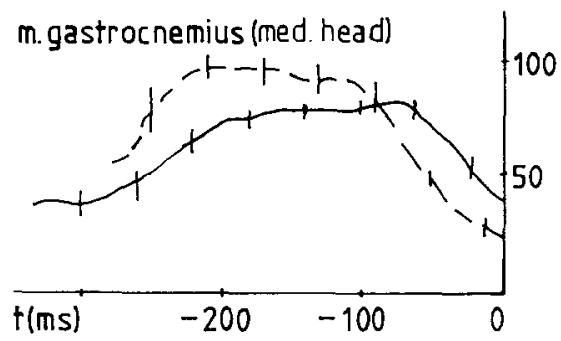

m. gastrocnemius (lat. head)
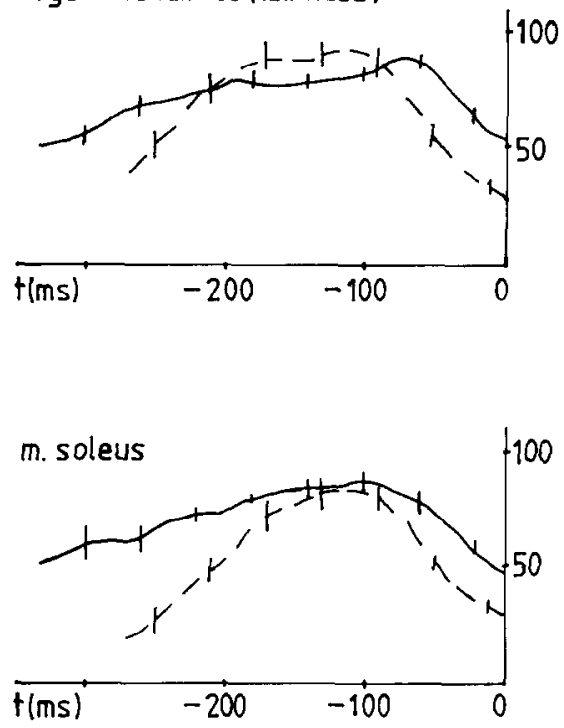

Fig. 3. Mean time histories of EMG-levels of both heads of $\mathrm{m}$. gastrocnemius and $\mathrm{m}$. soleus during CMJ and EKJ. EMG-levels are expressed in terms of percent of the maximum value attained during CMJ. Time $(t)$ is expressed relative to instant of toe-off $(t=0)$. Thin vertical bars indicate SEM. $n=11$.

Fig. 2 also shows mean time histories of the angle in the ankle $\left(\Theta_{\mathrm{A}}\right)$ and mean time histories of the angular velocities of plantar flexion for both jumps $\left(\dot{\Theta}_{\mathrm{A}}\right)$. Note that the angle is greater during EKJ than during CMJ, except for the last $20 \mathrm{msec}$. The angular velocity attains a maximum of $15.9 \pm 2.2 \mathrm{rad} / \mathrm{sec}$ during $\mathrm{CMJ}$, and a maximum of 


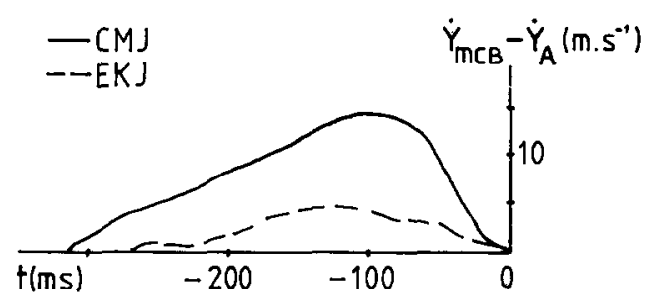

Fig. 4. Mean time histories of velocity difference between the mass center of body and the ankle $\left(\dot{Y}_{\mathrm{MCB}}-\dot{Y}_{\mathrm{A}}\right)$ during CMJ and EKJ. Time $(t)$ is expressed relative to instant of toe-off $(t=0)$.

$7.5 \pm 1.4 \mathrm{rad} / \mathrm{sec}$ during EKJ. The maximum is attained $35.5 \mathrm{msec}$ before toe-off in CMJ, and $56.4 \mathrm{msec}$ before toe-off in EKJ. As can be seen the moment rapidly declines when the angular velocity increases.

Fig. 2 finally shows mean time histories of net power output about the ankle $\left(P_{\mathrm{A}}\right)$. The power output reaches a peak value of $1404 \pm 292$ $\mathrm{W}$ at $51 \mathrm{msec}$ before toe-off during CMJ, and a peak value of only $852 \pm 167 \mathrm{~W}$ at $95 \mathrm{msec}$ before toe-off during EKJ. The work done during the push-off, obtained by integration of power with respect to time, is $130 \pm 18 \mathrm{~J}$ for CMJ and $76 \pm 20 \mathrm{~J}$ for EKJ.

Fig. 4 presents mean time time histories of the velocity difference between the mass center of body and ankle $\left(\dot{Y}_{\mathrm{MCB}}-\dot{Y}_{\mathrm{A}}\right)$. Rotation of the upper body about the hips explains why $\dot{Y}_{\mathrm{MCB}}-\dot{Y}_{\mathrm{A}}$ departs from
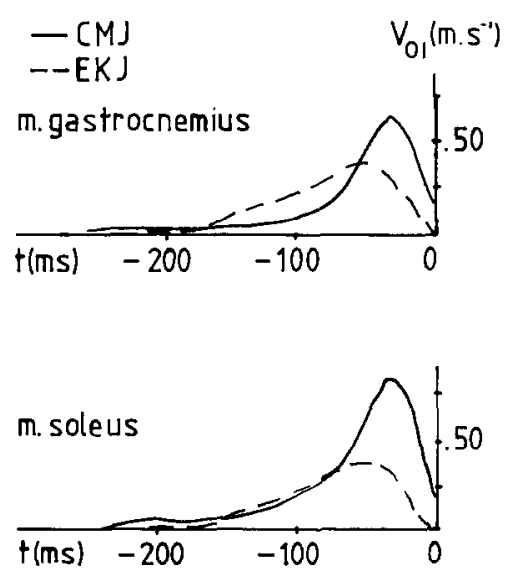

Fig. 5. Velocities with which origins of the muscles approach insertion $\left(V_{O I}\right)$ during CMJ and EKJ for $\mathrm{m}$. gastrocnemius and $\mathrm{m}$. soleus. Time $(t)$ is expressed relative to instant of toe-off $(t=0)$. 

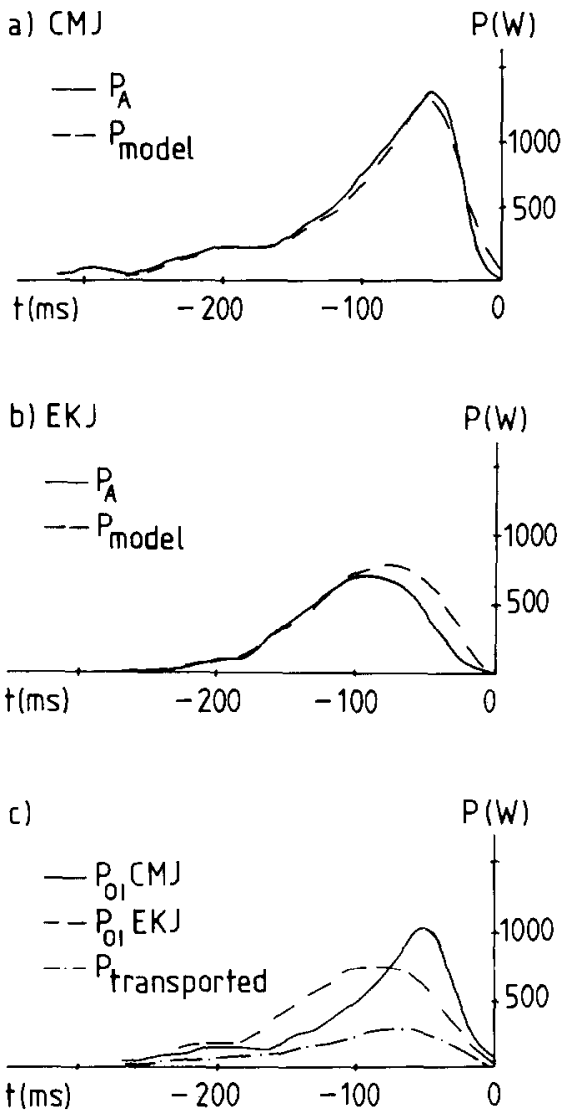

Fig. 6. Actual power output about the ankle $\left(P_{\mathrm{A}}\right)$ and the power output as predicted by the model ( $P_{\text {ruodel }}$ ) during CMJ (a) and EKJ (b). (c) The calculated power delivered by the contractile and elastic parts of the $\mathrm{m}$. triceps surea $\left(P_{O I}\right)$ during $\mathrm{CMJ}$ and EKJ, together with the power transported by $\mathrm{m}$. gastrocnemius from knee to ankle $\left(P_{\text {transported }}\right)$ in CMJ. Power transportation during EKJ was negligible and is not shown. Time $(t)$ is expressed relative to instant of toe-off $(t=0)$.

zero in EKJ. As can be seen $\dot{Y}_{\mathrm{MCB}}-\dot{Y}_{\mathrm{A}}$ reaches larger values and declines later, but more rapidly, in CMJ than in EKJ.

Fig. 5 shows mean time histories of $V_{O I}$ for $\mathrm{m}$. gastrocnemius and $\mathrm{m}$. soleus during both jumps.

Fig. 6 presents mean time histories of actual power output about the ankle $\left(P_{\mathrm{A}}\right)$ and power about the ankle calculated with help of the model $\left(P_{\text {model }}\right)$. The latter was obtained by multiplication of the mo- 


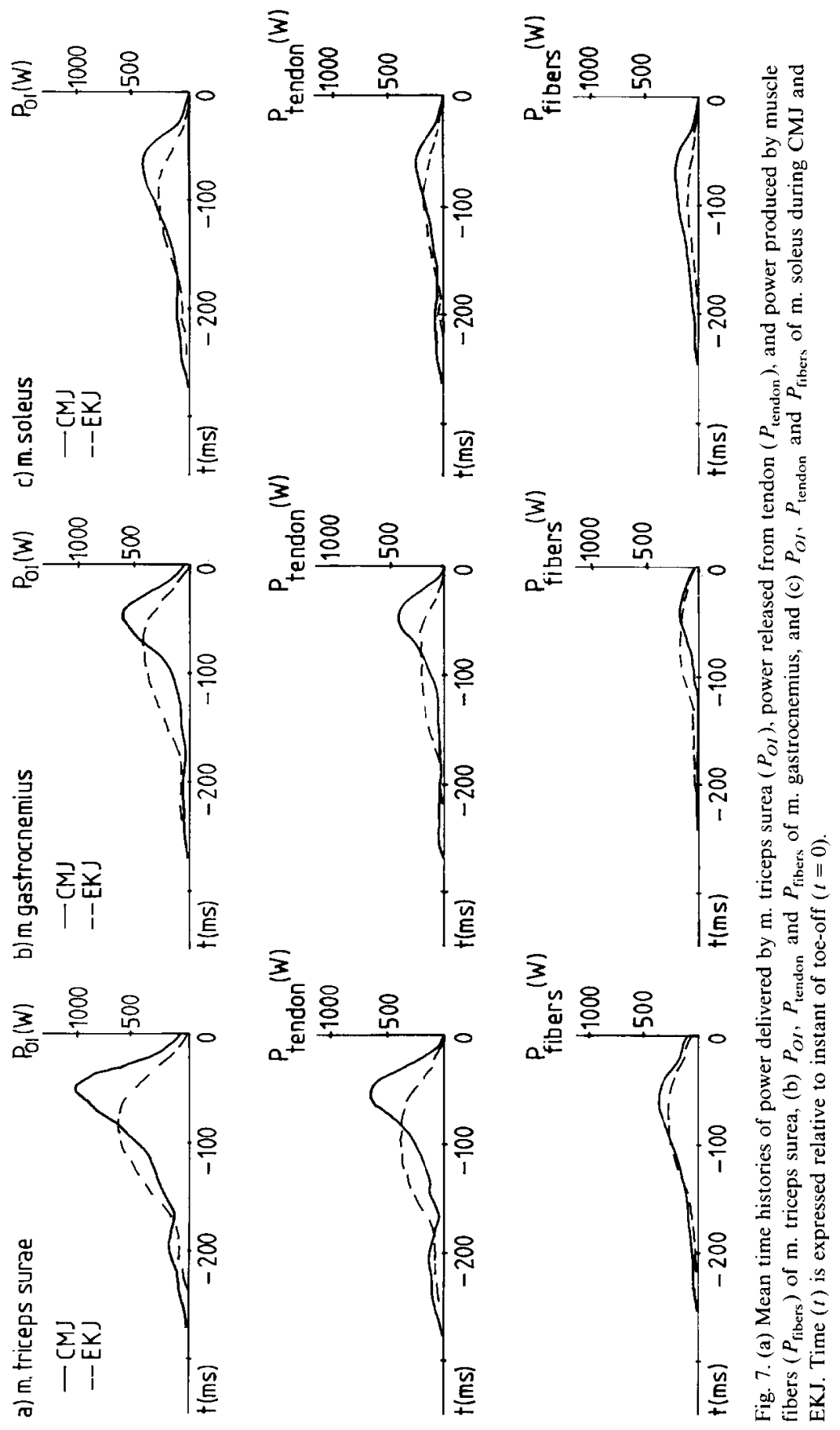


ment about the ankle calculated by the model and the angular velocity of plantar flexion. The curve of $P_{\text {model }}$ fits well to that of $P_{\mathrm{A}}$, both in CMJ (fig. 6a) and in EKJ (fig. 6b). Fig. 6c shows the calculated power delivered by the contractile and elastic parts of the $\mathrm{m}$. triceps surae $\left(P_{O I}\right)$ for CMJ and EKJ together. This figure also shows the power transported by the $\mathrm{m}$. gastrocnemius ( $\left.P_{\text {transported }}\right)$ from knee to ankle in CMJ. The power transported in EKJ was negligible and is not shown.

Fig. 7a presents for both jumps mean time histories of power delivered by $\mathrm{m}$. triceps surae $\left(P_{O I}\right)$, power released from tendon $\left(P_{\text {tendon }}\right)$ and power produced by muscle fibers $\left(P_{\text {fibers }}\right)$ of the $\mathrm{m}$. triceps surae. In fig. $7 \mathrm{~b}$ and fig. $7 \mathrm{c}$ the results are depicted for $\mathrm{m}$. gastrocnemius and $\mathrm{m}$. soleus respectively.

\section{Discussion}

In this study the mechanical output about the ankle during $\mathrm{CMJ}$ was compared with that during EKJ. The power output about the ankle was found to reach $1404 \mathrm{~W}$ in CMJ and $852 \mathrm{~W}$ in EKJ. The amount of work done about the ankle was also larger in CMJ than in EKJ. In CMJ it amounted $130 \mathrm{~J}$ and in EKJ $76 \mathrm{~J}$.

As stated in the introduction, one of the possible explanations for the difference in power output of the plantar flexors found in the ankle is pre-stretch potentiation, which might occur in CMJ but not in EKJ. But from fig. 2 it can be seen that $M_{\mathrm{A}}$ reaches the same value at the same (small) angular velocity. So there is no reason to assume that pre-stretch potentiation is responsible for some of the difference in power output between the two jumps.

Other possible explanations for the difference in power output about the ankle are transportation of power from knee to ankle via the bi-articular $\mathrm{m}$. gastrocnemius, and the catapult-phenomenon. It is necessary to use the calculations of the model of the $m$. triceps surae to find the contribution of these two mechanisms to the power output about the ankle. From fig. $6 \mathrm{a}$ and fig. $6 \mathrm{~b}$ it appears that for both jumps the model predicts quite well the output actually found. The discrepancy between the curves of mainly EKJ in the last part of the push-off may be attributed to the fact that in reality EMG-levels decrease (fig. 3), whereas the model presupposes a constant level of muscle excitation. Considering these findings, it seems acceptable to 
use the model calculations for comparing the output of tendon and muscle fibers of the $\mathrm{m}$. triceps surae during both jumps.

At first sight the only difference left between the two jumps is the absence of transportation of power in EKJ. If this were right the power output of $\mathrm{m}$. triceps surae should be the same in both jumps. But fig. $6 \mathrm{c}$ shows that this is not the case. The power delivered by $\mathrm{m}$. triceps surae $\left(P_{O I}\right)$ is greater in CMJ than in EKJ. So transportation of power is not the only explanation for the difference found in power output about the ankle. The remainder of the difference is caused by the more rapid release of energy in CMJ from predominantly $\mathrm{m}$. gastrocnemius tendon (catapult-action), as can be seen from fig. $7 \mathrm{~b}$. The rate of decrease of force is important: the faster this rate, the faster the tendinous structures shorten. It can be seen in fig. 2 that the rate of decrease in moment (and thus in force) is greater in CMJ than in EKJ. This faster decrease of force in CMJ is caused by a faster increase of $V_{O I}$ of $\mathrm{m}$. triceps surae. The reason for this faster increase of $V_{O I}$ is the faster increase of $\dot{\Theta}_{\mathrm{A}}$ during CMJ (fig. 2), which is caused by two factors. The first has to do with the influence of hip extension and knee extension on the vertical acceleration of $\mathrm{MCB}$ with respect to the ankle. In fig. 4 the vertical velocity difference between $\mathrm{MCB}$ and ankle $\left(\dot{Y}_{\mathrm{MCB}}-\dot{Y}_{\mathrm{A}}\right)$ is depicted for both jumps. The rate of decrease of $\dot{Y}_{\mathrm{MCB}}-\dot{Y}_{\mathrm{A}}$ is greater during CMJ than during EKJ as a result of a difference in the contribution of hip extension and knee extension. This causes a greater rate of decrease of external moment about the ankle (the moment working against the muscle moment) during CMJ. The second, less important, factor is the fact that $\Theta_{\mathrm{A}}$ is greater during EKJ than during CMJ (fig. 2), so the moment delivered by the plantar flexors can add less to the acceleration of MCB. The transformation of muscular output to vertical energy of the body's mass center is thus less effective (Van Ingen Schenau et al. 1987).

This is an interesting observation. It demonstrates the interdependency of movement dynamics and muscular power output. It is not only the muscular capacity to deliver power that determines the dynamics of a movement, but the movement dynamics also determine how much power can be delivered by muscles.

The catapult phenomenon helps to explain the difference in power output about the ankle between CMJ and EKJ, but not the difference in work output. It is clear that the work output about the ankle during $\mathrm{CMJ}$ is greater than during EKJ. A possible explanation is that a part 
of the angular displacement of the ankle is travelled at submaximal moment. But from fig. 2 it can be seen that when $M_{\mathrm{A}}$ is maximal, only a little change in $\Theta_{\mathrm{A}}$ has occurred. So this factor can be excluded as explanation for a part of the difference in work output found in the ankle.

During CMJ there is transportation of energy via the bi-articular $\mathrm{m}$. gastrocnemius. This explains a part of the difference in work output between CMJ and EKJ. The remainder of the difference is the result of the fact that $\mathrm{m}$. soleus exerts force over a greater external contraction range during $\mathrm{CMJ}(6.1 \mathrm{~cm})$ than during $\mathrm{EKJ}(3.4 \mathrm{~cm})$. The contraction range of $\mathrm{m}$. gastrocnemius is $3.8 \mathrm{~cm}$ during $\mathrm{CMJ}$ and $3.5 \mathrm{~cm}$ during EKJ, but m. gastrocnemius does not deliver more work during the push-off in CMJ, because the force of this muscle is predominantly lower in CMJ.

Summarizing, it may be stated that there is no reason to assume that the contractile properties differ between CMJ and EKJ as the result of pre-stretch potention. The difference in power output between CMJ and EKJ is explained by a greater contribution of transportation of power from knee to ankle via $\mathrm{m}$. gastrocnemius and catapult-action in CMJ. The difference in work done about the ankle is, in addition to transportation of energy, also determined by a difference in contraction range of $\mathrm{m}$. soleus.

\section{References}

Alexander, R. McN. and H.C. Bennet-Clark, 1977. Storage of elastic strain energy in muscles and other tissues. Nature 265, 114-117.

Basmajian, J.V., 1978. Muscles alive: their functions revealed by electromyography. Baltimore: Williams and Wilkins.

Bobbert, M.F., P.A. Huijing and G.J. van Ingen Schenau, 1986. A model of the human triceps surae muscle-tendon complex applied to jumping. Journal of Biomechanics 19, 887-898.

Bobbert, M.F., G.J. van Ingen Schenau and P.A. Huijing, 1985. 'Plantar flexion torques in jumping and in isokinetic plantar flexion, a comparison'. In: S.M. Perren and E. Schneider (eds.), Biomechanics: current interdisciplinary research.

Cavagna, C.A., 1978. 'Effects of efficiency and inefficiency of terrestrial locomotion'. In: E. Asmussen and K. Jorgensen (eds.), Biomechanics VI-A. International series on biomechanics, Vol 2A. Baltimore: University Park Press. pp. 3-22.

Elftman, H., 1939. Forces and energy changes in the leg during walking. American Journal of Physiology 125, 339-356.

Fugl-Meyer, A.R., K.H. Mild and J. Horn Sten, 1982. Output of skeletal muscle contractions: a study of isokinetic plantar flexion in athletes. Acta Physiologica Scandinavia 115, 193-199. 
Gregoire, L., H.E. Veeger, P.A. Huijing and G.J. van Ingen Schenau, 1984. Role of mono- and biarticular muscles in explosive movements. International Journal of Sports Medicine 5, 301-305.

Grieve, D.W., S. Pheasant and P.R. Cavanagh, 1978. 'Prediction of gastrocnemius length from knee and ankle joint posture'. In: E. Asmussen and K. Jorgensen (eds.), Biomechanics VI-A. International series on biomechanics, Vol 2A. Baltimore: University Park Press. pp. 405-412.

Hof, A.L., B.A. Geelen and J.W. van den Berg, 1983. Calf muscle moment, work and efficiency in level walking: role of series elasticity. Journal of Biomechanics 16, 523-537.

Komi, P.V. and P.R. Cavanagh, 1977. Electromechanical delay in human skeletal muscle. Medicine and Science in Sports and Exercise 9, 49.

Van Ingen Schenau, G.J., 1984. An alternative view of the concept of utilisation of elastic energy in human movement. Human Movement Science 3, 301-336.

Van Ingen Schenau, G.J., M.F. Bobbert, P.A. Huijing and R.D. Woittiez, 1985. The instantaneous torque-angular velocity relation in plantar flexion during jumping. Medicine and Science in Sports and Exercise 17, 422-426.

Van Ingen Schenau, G.J., M.F. Bobbert and R.H. Rozendal, 1987. The unique function of the biarticular m. gastrocnemius in jumping. Journal of Anatomy (in press).

Van Soest, A.J., M.E. Roebroeck, M.F. Bobbert, P.A. Huijing and G.J. van Ingen Schenau, 1985. A comparison of one-legged and two-legged countermovement jumps. Medicine and Science in Sports and Exercise 17, 635-639.

Woittiez, R.D., R.H. Rozendal and P.A. Huijing, 1985. 'The functional significance of architecture of the human triceps surae muscle'. In: D.A. Winter et al. (ed.), Biomechanics IX. International series on biomechanics, Vol 5A. Champaign, IL: Human Kinetic Publishers. pp. 31-26. 